\title{
A method to determine pressure distribution of the hand
}

\author{
R PREM KUMAR* \& J W BRANDSMA $\dagger$ \\ * Schieffelin Le prosy Research and Training Center, Karigiri South \\ India-632106 †Rehabilitation Research Department, National \\ Hansen's Disease Center, USPHS Hospital, Carville, LA 70721, \\ USA
}

Accepted for publication 5 September 1985

\begin{abstract}
Summary A simple method to determine pressure distribution, and to identify areas of localized high pressure of the deformed and often insensitive hand is presented. The method will also be useful to evaluate tool adaptations and the effect of surgery on the contact bearing area of the hand.
\end{abstract}

\section{Introduction}

There are 3 methods to measure the distribution of pressure on hands and feet. The identification of areas of localized high pressure is especially important in the management of deformed hands and feet which very often are also insensitive. Once areas of high pressure have been identified, tool adaptations and modified footwear will help to prevent recurrence of ulcers.

In one method force transducers are used. This method requires expensive electronic instrumentation and therefore is not practical to use in leprosy hospitals when we are dealing with large numbers of patients. Time, money and expertise are also usually not available. In another method the so-called slipper socks* or gloves are used. The gloves and socks are lined with pressure sensitive capsules that contain different coloured dyes that burst at specific pressures and thus will stain the glove or sock.

The third method which is the most practical uses the Harris mat. $\dagger$ This rubber mat contains ridges of graduated heights which form squares of varying size. Light pressure will be revealed in the print as large squares. With heavier

* Slipper socks available from: National Hansen's Disease Center, USPHS Hospital, Vocational Rehabilitation Department, Carville, LA 70721, USA.

† Harris mats available from: Downs Surgery, Inc. 2500 Park Central Blvd. Decatur, GA 30035 and Apex Foot Products, 330 Philips Avenue, South Hackensack, NJ 07601. 
pressure the deeper ridges of the smaller squares will also be printed on the paper and the print will be darker. For the foot we can make a static print where the patient is just asked to step on the mat. We can obtain a dynamic print when we ask the patient to step on the mat when he takes a few steps in the room, or we can put a piece of Harris mat the size of his shoe within the shoe and ask him to take a few steps.

As far as we know the Harris mat has only been used to assess pressure distribution of the foot. We feel that these mats can also be used to assess pressure distribution of the hands. It is especially suitable to assess the contact bearing area of the hand in a cylindrical grip. Most of the farming tools will require such a grip. The different types of pinch cannot be evaluated with this method.

\section{Method}

A piece of Harris mat, the size of the circumference of the cylinder, is cut from a larger mat. The mat is then glued onto the cylinder. In our case the diameter of the cylinder was 2 inches. Wooden cylinders with different diameters and also Harris mats of different thicknesses can be used. The best would be to use a cylinder which is the size of the common farming tools used in your area. You could also have samples of the common tools in your department with a piece of Harris mat wrapped around them. 'Velcro' or rubber band can be used at the edges of the mat if you do not want to fix it permanently to the cylinder or tool.

The mat is inked using black printers ink (Figure 1), then a piece of absorbent paper is placed around the mat. The surface is rubbed gently with cotton wool to spread the ink evenly and remove any excess of ink (Figure 2). A piece of paper the size of the cylinder is then carefully wrapped around the cylinder. Only the edges

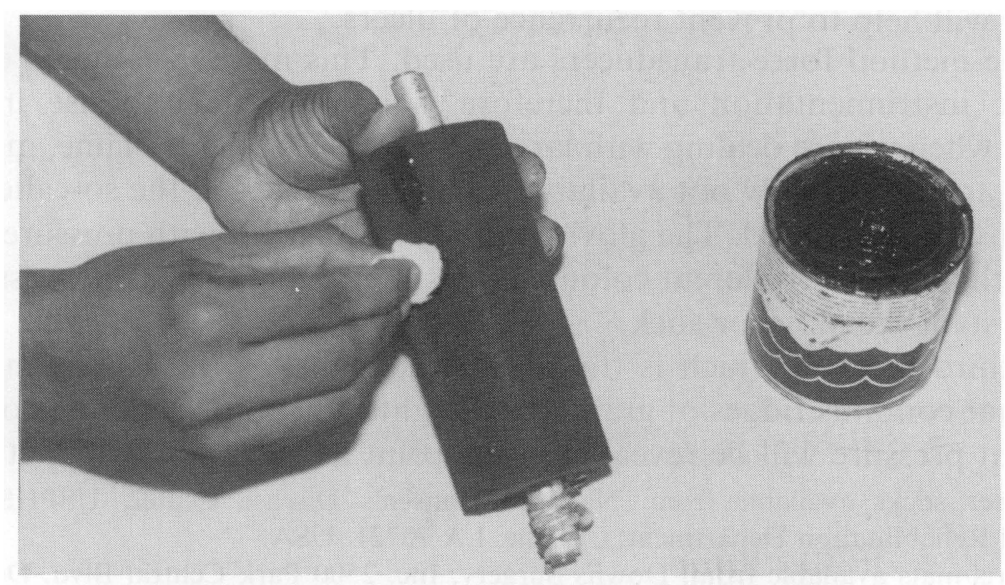

Figure 1. Application of printers ink to a Harris mat. 


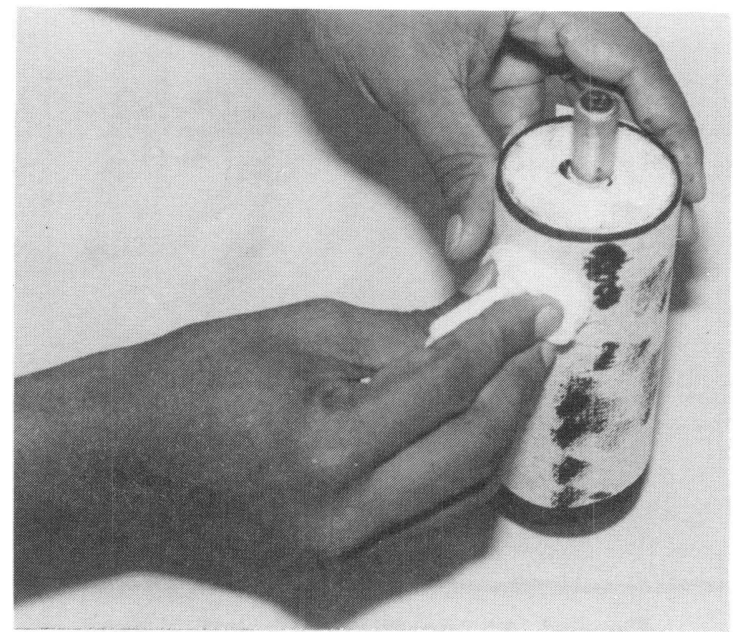

Figure 2. Even distribution of ink and removal of excess ink.

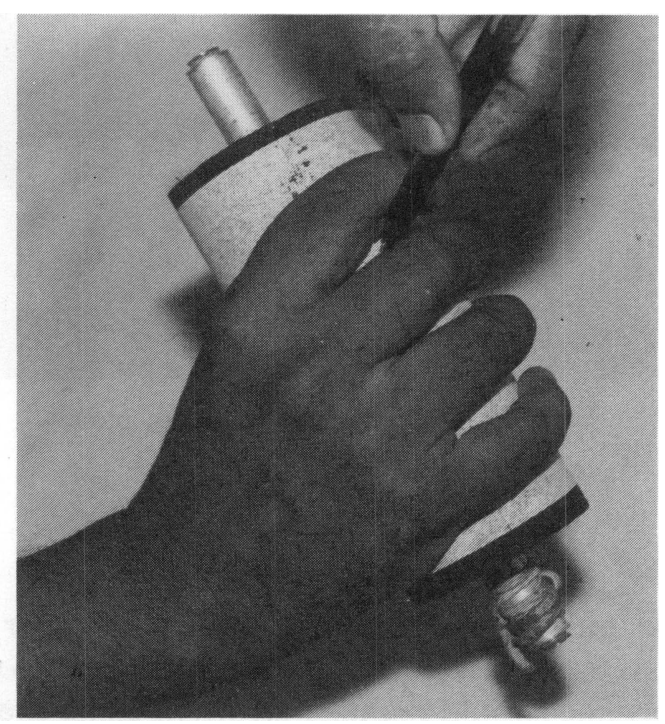

Figure 3. Tracing the outline of a hand.

should be touched in order not to leave prints on the paper. The patient is then asked to grasp the cylinder firmly. The outline of the hand is then traced with a pen so that after removal of the paper it will be easy to accurately localize the areas of high pressure (Figure 3). Prints of a normal hand and a claw hand are shown in Figures 4 and 5. 


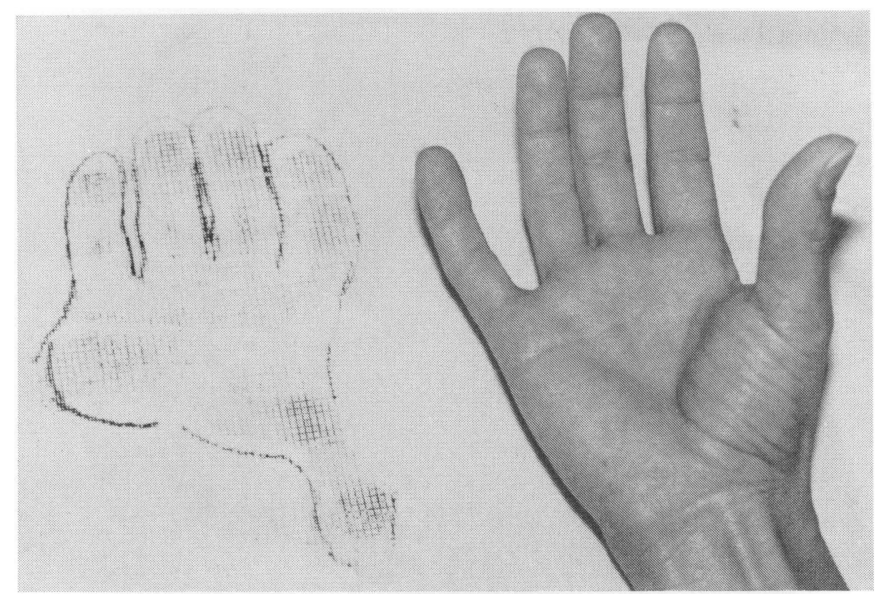

Figure 4. An impression of a normal hand.

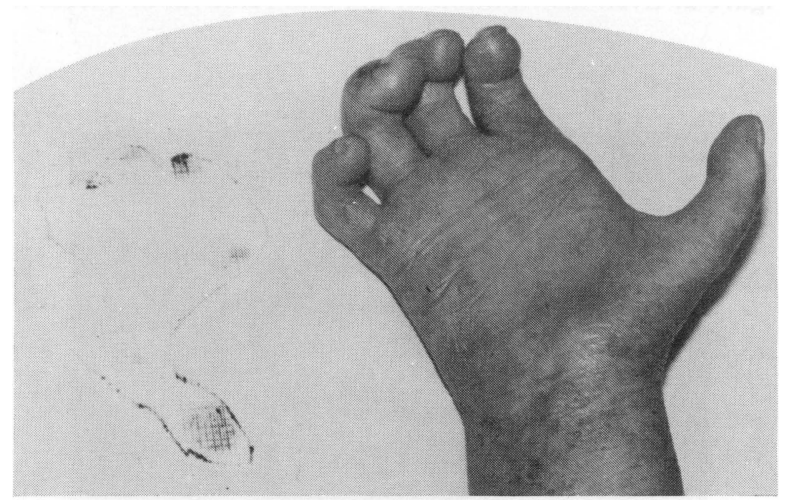

Figure 5. An impression of a claw hand.

\section{Discussion}

It is difficult with this method to measure the effect of shear forces on the hand. The glove method that uses pressure sensitive micro capsules would be superior in this regard. However, one should realize that when using the gloves there should be a glove that fits well for every hand. This would make this method expensive to use in developing countries. The glove could also to some extent already correct certain deformities.

The Harris mat method is easier to use and will identify areas of localized high pressure, though in a static grip. The areas of high pressure may increase and change when the hand is at work. It is not wise to wait and see when and where injuries may occur, when it is possible to determine what the potential danger areas are. The patient can then be advised about working habits and tool adaptations can be made. 
This method can also be very useful to evaluate the contact bearing area of the hand pre- and postoperatively in conjunction with tendon transfer surgery. The method does not quantitate the pressure but gives a good clinical impression of the area of the hand that is in contact with the tool and will clearly show areas with increased pressure.

A cylindrical object of glass or plastic from the laboratory is also a good tool to assess the contact bearing area of the hand. The patient is asked to grasp this object and the examiner will be able to see through the cylinder the areas of the hand that are in contact with the cylinder. These areas will show more white as blood will be pressed from them.

One of us has also used a grip index to evaluate grip contact pre- and postoperatively in conjunction with tendon transfer surgery. The fingers were given a maximum grade of 3 , one for each phalanx, and the thumb a maximum grade of 2 (two phalanges). The number of phalanges that were in contact with the cylinder were written down. A normal hand would, therefore, have a grip index of 2-3-3-3-3. A claw hand could have a grip index of 2-3-3-1-0. This would indicate that the thumb, index, and middle finger were in full contact with the cylinder, that only the distal phalanx of the ring finger was in contact and that the little finger was without any contact. Using this method we were able to show that there was an increased grip contact postoperatively. With these 2 methods, however, we will only be able to record the area of the hand that is in contact with the cylindrical object. Localized areas of high pressure can only be identified with the Harris mat method.

\section{Acknowledgment}

We wish to thank Dr P W Brand who suggested this method of pressure assessment to us.

\section{Selected bibliography}

${ }^{1}$ Brand PW. Repetitive stress on insensitive feet. The pathology and management of plantar ulceration in neuropathic feet. USPHS Hospital, Carville, LA 70721, USA.

${ }^{2}$ Brand PW, Ebner JD. A pain substitute. Pressure assessment in the insensitive limb. Am J Occ Ther, 1969; 23: 479-86.

${ }^{3}$ Brand PW, Ebner JD. The 'Slipper-sock' footprint test. USPHS Hospital, Carville, LA 70721, USA.

${ }^{4}$ Brand PW, Ebner JD. Pressure sensitive devices for denervated hands and feet. J Bone \& Joint Surg, 1969; 51A: 109-16.

${ }^{5}$ Brandsma JW, Watson JM. Clawfinger correction. Results of extensor-flexor many tailed operation. The Hand, 1982; 14: 307-12.

${ }^{6}$ Corson M, 'Blue Prints' warn the handicapped and aid the disabled. The Star, 1970; 29 (4); 7-10.

7 Shipley DE. The footprint test, USPHS Hospital, Carville, LA 70721, USA. 